\title{
Efeitos do Manejo Pós-Parto de Vacas Primíparas no Desempenho de Bezerros de Corte até Um Ano de Idade ${ }^{1}$
}

\section{Bernardo Augusto Albornoz Pötter ${ }^{2}$, José Fernando Piva Lobato ${ }^{3}$, Flávio Schramm Schenkel ${ }^{4}$}

\begin{abstract}
RESUMO - Os efeitos no desenvolvimento de bezerros submetidos a: a) três tratamentos, consitindo de cargas animais pós-parto (CA) de $240 \mathrm{~kg}$ de peso vivo (PV)/ha (tratamento 1, T1), de $320 \mathrm{~kg}$ de PV/ha (T2), ambas em campo nativo, e de $400 \mathrm{~kg}$ de PV/ha em pastagem melhorada de azevém (Lolium multiflorum L.) por 80 dias pós-parto e, posteriormente, em CA igual à do T2 em campo nativo (T3); b) duas idades de desmame, consistindo de desmame aos 100 (DP) ou 180 (DC) dias de idade, foram avaliados utilizando 92 bezerros filhos de vacas primíparas Hereford e Braford. O sexo dos bezerros influenciou significativamente o peso ao nascer, não sendo observado efeito da raça da vaca. Os tratamentos e a raça da vaca não influenciaram o ganho médio diário (GMD) dos bezerros do nascimento ao desmame precoce. Bezerros filhos de vacas Braford tiveram maiores pesos no DP (PDP) que os filhos de vacas Hereford, enquanto os tratamentos não influenciaram o PDP. Os bezerros filhos de vacas Braford no T2 tiveram GMD até os 180 dias (GMD180) e PV aos 180 dias (PAJ180) superiores aos bezerros filhos de vacas Braford no T1 e Hereford no T2. Os bezerros do DP tiveram GMD180 e PAJ180 significativamente inferiores aos bezerros do DC. Entretanto, não se verificaram efeitos de tratamentos e idade de desmame sobre o GMD até os 365 dias e o peso ajustado aos 365 dias de idade. Portanto, os três manejos pós-parto das vacas e as duas idades de desmame dos bezerros avaliados não influenciaram o desenvolvimento dos bezerros a um ano de idade.
\end{abstract}

Palavras-chave: bezerros de corte, carga animal, ganho de peso, idade de desmame

\section{Effects of Post-Partum Management of Primiparous Cows on Growth of Beef Calves Up to One Year of Age}

\begin{abstract}
The effects on the performance of calves submitted to: a) three treatments, consisted of post-partum stocking rates (CA) of $240 \mathrm{~kg}$ of live weight (LW)/ha (treatment 1, T1), of $320 \mathrm{~kg}$ of LW/ha (T2), both on natural pastures, and of $400 \mathrm{~kg}$ of LW/ha on improved pasture of Italian ryegrass (Lolium multiflorum L.) during the first 80 days post-partum, and after on natural pasture at the same stocking rate as T2 afterwards (T3); b) two weaning ages, consisted of weaning at 100 (EW) and at 180 (CW) days of age, were evaluated using 92 beef calves from Hereford and Braford primiparous cows. The sex of the calves affected significantly the birth weight, and no effect was observed for the breed of cow. The treatments and breed of cow did not affect the average daily gain (ADG) of the calves from birth to early weaning (GEW). Calves born from Braford cows had higher LW at EW (WEW) than calves born from Hereford cows, while the WEW was not affected by the treatments. Calves born from Braford cows on T2 had ADG up to 180 days (ADG180) and LW at 180 days (LW180) greater than calves born from Braford cows on T1 and Hereford cows on T2. Calves from CW had greater LW180 and ADG180 than calves from EW. However, treatments and weaning ages did not affect the LW at 365 days and the ADG up to 365 days of age. The three cows post-partum management schemes and the two calves weaning ages evaluated did not affect the calves performance up to one year of age.
\end{abstract}

Key Words: beef calves, liveweight gain, stocking rate, weaning age

\section{Introdução}

A produtividade de um sistema pecuário na fase de cria, avaliada pelos quilos de bezerro desmamado/ unidade de superfície, é o resultado da fertilidade das vacas e do peso dos bezerros ao desmame (Simeone \& Lobato, 1998). O nível nutricional do rebanho de cria, no pré e pós-parto, influencia a fertilidade das vacas e influi no peso dos bezerros ao desmame por meio do seu efeito sobre a produção de leite das vacas (Quadros \& Lobato, 1997; Lobato et al., 1998). Segundo Espasandin et al. (2001), a herdabilidade média para produção de leite em gado de corte é da ordem de 0,23 a 0,41 , com grande influência dos

\footnotetext{
${ }^{1}$ Parte da Dissertação de Mestrado do primeiro autor.

${ }^{2}$ Médico Veterinário, Mestre em Zootecnia - UFRGS - Porto Alegre - RS. E.mail: bpotter@bol.com.br

3 Professor Adjunto IV, Departamento de Zootecnia - UFRGS, Bolsista do CNPq. Porto Alegre - RS, C. Postal 776 - 90001-970.

E.mail: lobato@orion.ufrgs.br

${ }^{4}$ Pesquisador Associado, University of Guelph, Canadá. E.mail: schenkel@uoguelph.ca
} 
fatores ambientais. Desse modo, o nível de oferta de forragem ao qual o rebanho de cria é submetido influencia a produção de leite das vacas e o desempenho dos bezerros até a desmama.

Quadros \& Lobato (1997) trabalharam com vacas primíparas e observaram ter as vacas submetidas à carga animal em campo nativo de $240 \mathrm{~kg} \mathrm{PV} / \mathrm{ha}$ $(0,6 \mathrm{EV} / \mathrm{ha})$ maior produção de leite $(6,39 \mathrm{~kg}$ de leite/ vaca/dia) em relação às vacas mantidas em carga animal de $320 \mathrm{~kg} \mathrm{PV} / \mathrm{ha}(0,8 \mathrm{EV} / \mathrm{ha})(5,52 \mathrm{~kg}$ de leite/ $\mathrm{vaca} / \mathrm{dia})$. Os bezerros filhos das vacas do tratamento $0,6 \mathrm{EV} /$ ha tiveram maior ganho médio diário (GMD; $0,768 \mathrm{~kg} / \mathrm{dia}$ ) e foram significativamente mais pesados $(161 \mathrm{~kg})$ ao desmame, quando comparados aos bezerros filhos de vacas do tratamento $0,8 \mathrm{EV} / \mathrm{ha}$ $(0,676 \mathrm{~kg} / \mathrm{dia}$ e $148 \mathrm{~kg}$, respectivamente). Simeone \& Lobato (1998) também observaram maiores GMD e pesos ao desmame de bezerros filhos de vacas primíparas submetidas a cargas animais de $0,60 \mathrm{EV} / \mathrm{ha}$ e $0,85 \mathrm{EV} / \mathrm{ha}$, sendo $0,720 \mathrm{~kg} / \mathrm{dia}$ e $158,7 \mathrm{~kg}$ para os primeiros e $0,677 \mathrm{~kg} / \mathrm{dia}$ e $148,9 \mathrm{~kg}$ para os últimos, respectivamente.

A raça ou cruzamentos também podem influenciar a produção de leite das vacas. Vários autores têm relatado uma produção de leite e maiores pesos de bezerros ao desmame por parte das vacas cruzadas em relação às vacas de raça pura (Cundiff et al., 1974; Cartwright, 1976; Lamond, 1976; Gregory et al., 1992). Ribeiro \& Lobato (1988) avaliaram o desempenho de bezerros filhos de vacas primíparas de três grupos raciais: G1- 3/4 Red Angus, 1/4 Devon; G2-3/4 Charolês, 1/4 Devon; G3- 1/2 Tabapuã, 1/2 Devon. O ganho de peso dos bezerros até o desmame e o peso ao desmame foi significativamente superior para os bezerros filhos de vacas do G3, cruza taurino $x$ zebuíno, em relação aos demais, cruza taurino $\mathrm{x}$ taurino. Barcellos \& Lobato (1992a; b) também constataram ter os bezerros cruza Nelore x Hereford, tanto nascidos na primavera quanto no outono, maiores ganhos de peso pré-desmama e maiores pesos vivos ao desmame.

Embora o desmame precoce seja eficiente em incrementar a fertilidade das vacas, o desenvolvimento dos bezerros desmamados precocemente é dependente do manejo alimentar que os mesmos recebem após o desmame. No Rio Grande do Sul, diversos trabalhos foram realizados envolvendo a utilização de pastagens melhoradas ou campo nativo com e sem suplementação após o desmame precoce para avaliar o desenvolvimento dos bezerros (Moraes
\& Lobato, 1993; Simeone \& Lobato, 1998; Lobato et al., 1999; Marques et al., 2001a; Costa et al., 2002).

O presente trabalho foi conduzido com o objetivo de avaliar o desenvolvimento de bezerros de corte filhos de vacas primíparas Hereford e Braford, submetidas à duas cargas animais em campo nativo e em pastagem nativa melhorada com azevém durante 80 dias pós-parto, desmamados aos 100 ou 180 dias de idade, até os 365 dias de idade.

\section{Material e Métodos}

O experimento foi conduzido na Agropecuária Caty, localizada no segundo sub-distrito de Quaraí, na fronteira oeste do Rio Grande do Sul. A vegetação predominante é o capim-caninha (Andropogon lateralis) com ocorrências de capim melador (Paspalum dilatatum), grama forquilha (Paspalum notatum) e pega-pega (Desmodium incanum). Foram utilizadas 92 vacas primíparas aos três anos de idade, das raças Braford e Hereford, distribuídas aleatoriamente em três tratamentos de manejo pós-parto:

$\mathrm{T} 1$ = Carga animal (CA) de $240 \mathrm{~kg}$ de peso vivo $(\mathrm{PV}) / \mathrm{ha}$ em campo nativo $(0,6 \mathrm{EV} / \mathrm{ha}-\mathrm{EV}=$ equivalente vaca $=400 \mathrm{~kg} \mathrm{PV}$; Cocimano et al., 1983);

$\mathrm{T} 2=\mathrm{CA}$ de $320 \mathrm{~kg}$ de $\mathrm{PV} / \mathrm{ha}$ em campo nativo $(0,8 \mathrm{EV} / \mathrm{ha})$;

$\mathrm{T} 3=\mathrm{CA}$ de $400 \mathrm{~kg}$ de PV/ha (1 EV/ha) em pastagem melhorada de azevém (Lolium multiflorum L.) durante 80 dias pós-parto, seguindo para campo nativo com carga animal igual ao tratamento $\mathrm{T} 2$.

Em cada tratamento de manejo pós-parto, $50 \%$ das vacas eram Hereford e 50\% eram Braford. Em 01.01.01, foi realizado o desmame de 50\% dos bezerros (as) de cada tratamento. Ou seja, os seguintes tratamentos passaram a fazer parte do experimento:

Desmame precoce $(\mathrm{DP})=46$ bezerros (as) desmamados em média com 100 dias de idade;

Desmame convencional $(\mathrm{DC})=46$ bezerros (as) desmamados em média com 180 dias de idade.

Os bezerros do DP permaneceram em mangueira por cinco dias pós-desmama, com água e concentrado com $18 \%$ de proteína bruta (PB), no nível de $1 \%$ do peso vivo. Após o sexto dia, foram para potreiros de pastagem melhorada de cornichão (Lotus corniculatus cv. São Gabriel) recebendo o mesmo concentrado até a data do DC, o qual foi feito em 06.03.2001, quando os bezerros tinham em média 180 dias de idade. A partir desta data, os bezerros foram manejados sobre resteva de lavoura de arroz, com-

R. Bras. Zootec., v.33, n.2, p.426-433, 2004 
posto basicamente por rebrote de arroz (Oryza sativa) e capim-arroz (Echinochloa column), na carga animal de $243 \mathrm{~kg} \mathrm{PV} / \mathrm{ha}$ até 09.08.2001, quando as fêmeas foram para um potreiro de campo nativo e os machos para um potreiro de pastagem de azevém. Os bezerros foram pesados a cada 28 dias mediante jejum prévio de 12 horas.

O experimento foi analisado segundo um delineamento completamente casualizado em arranjo fatorial. Todas as variáveis-resposta foram analisadas mediante análise de variância, considerando o número desigual de repetições. Para múltiplas comparações entre médias ajustadas foi utilizado o teste Tukey.

O peso ao nascer (PN), o ganho médio diário até o desmame precoce (GNP) e o peso na data do desmame precoce (PDP) foram analisados segundo o seguinte modelo:

$$
\mathrm{Y}_{\text {hikl }}=\mathrm{u}+\mathrm{G}_{\mathrm{h}}+\mathrm{T}_{\mathrm{i}}+\mathrm{S}_{\mathrm{k}}+\mathrm{GxT}_{\mathrm{hi}}+\mathrm{b}_{1} \mathrm{DJP}+\mathrm{e}_{\mathrm{hikl}}
$$

em que $\mathrm{Y}_{\text {hikl }}=$ observação do PN, GNP ou PDP realizada no l-ésimo terneiro, do k-ésimo sexo, do i-ésimo tratamento e da h-ésima composição racial da vaca; $u=$ média geral; $G_{h}=$ efeito fixo da h-ésima raça da vaca, $h=1,2 ; \mathrm{T}_{\mathrm{i}}=$ efeito fixo do i-ésimo tratamento, $\mathrm{i}=1,2$ e $3 ; \mathrm{S}_{\mathrm{k}}=$ efeito fixo do k-ésimo sexo, $\mathrm{k}=1,2 ; \mathrm{GxT}_{\mathrm{hi}}=$ efeito fixo da interação entre e a h-ésima raça e o i-ésimo tratamento; DJP = data juliana do parto; $b_{1}=$ coeficiente de regressão linear na data juliana de parto; $\mathrm{e}_{\text {hikl }}=$ efeito residual aleatório associado à observação $y_{h i k l}$.

O ganho médio diário até os 180 dias (GMD180) e até os 365 dias (GMD365) e os pesos ajustados aos 180 (PAJ180) e 365 (PAJ365) dias foram analisados segundo o seguinte modelo:

$$
\begin{gathered}
Y_{h i j k l}=u+G_{h}+T_{i}+D_{j}+T_{x D} D_{i j}+G_{h i}+ \\
G_{h x D}+G_{h x D} D_{h i j}+S_{k}+b_{1} D J P+e_{h i j k l}
\end{gathered}
$$

em que $Y_{\text {hijkl }}=$ observação do GMD180, PAJ180, GMD365 ou PAJ365 realizada no l-ésimo terneiro, do k-ésimo sexo, pertencente à j-ésima época de desmame, do i-ésimo tratamento e da h-ésima composição racial da vaca; $u=$ média geral; $G_{h}=$ efeito fixo da g-ésima raça da vaca, $\mathrm{h}=1,2 ; \mathrm{T}_{\mathrm{i}}=$ efeito fixo do i-ésimo tratamento, $\mathrm{i}=1,2 \mathrm{e} 3 ; \mathrm{D}_{\mathrm{j}}=$ efeito fixo da $\mathrm{j}$-ésima idade de desmame, $\mathrm{j}=1,2 ; \mathrm{TxD}_{\mathrm{ij}}=$ efeito fixo da interação entre o i-ésimo tratamento e a j-ésima época de desmame; $\mathrm{GxT}_{\mathrm{hi}}=$ efeito fixo da interação entre e a h-ésima raça e o i-ésimo tratamento;
$\mathrm{GxD}_{\mathrm{hj}}=$ efeito fixo da interação entre e a h-ésima raça e a j-ésima época de desmame; $\mathrm{GxTxD}_{\mathrm{hij}}=$ efeito fixo da interação entre a h-ésima raça, i-ésimo tratamento, e a j-ésima época de desmame; $\mathrm{S}_{\mathrm{k}}=$ efeito fixo do k-ésimo sexo, $\mathrm{k}=1,2$; DJP = data juliana do parto; $b_{1}=$ coeficiente de regressão linear na data juliana de parto; $\mathrm{e}_{\mathrm{hijkl}}=$ efeito residual aleatório associado à observaçã̃o $\mathrm{y}_{\text {hijkl }}$.

\section{Resultados e Discussão}

O sexo dos bezerros influenciou $(\mathrm{P}<0,01)$ o $\mathrm{PN}$. Os bezerros machos tiveram $(\mathrm{P}<0,01)$ maiores $\mathrm{PN}$ $(36,3 \mathrm{~kg})$ que as fêmeas $(32,8 \mathrm{~kg})$ (Tabela 1). Os maiores PN dos machos em relação às fêmeas têm sido relatados por alguns autores (Holland et al., 1977; Lobato et al., 1998; Sakaguti et al., 2001). Holland et al. (1977), na Austrália, observaram ser os machos 5\% mais pesados ao nascer em relação às fêmeas. Lobato et al. (1998) citam diferenças de PN entre machos e fêmeas da ordem de $7,2 \%$ a favor dos bezerros machos, semelhante aos $6,9 \%$ de superioridade no $\mathrm{PN}$ dos machos em relação às fêmeas relatados por Sakaguti et al. (2001). Entretanto, esses valores são inferiores aos observados no presente trabalho, onde os machos foram $10,6 \%$ mais pesados ao nascer em relação às fêmeas.

De acordo com a distribuição aleatória das vacas nos tratamentos (randomização), não houve diferença ( $\mathrm{P}>0,05)$ entre as médias do $\mathrm{PN}$ dos bezerros dos três tratamentos (Tabela 1).

Não foram observados efeitos $(\mathrm{P}>0,05)$ da interação raça da vaca $x$ tratamento, para as variáveis PN, GDP e PDP. Os valores variaram de 33,3 a $35,4 \mathrm{~kg} ; 0,808$ a $1,0 \mathrm{~kg} /$ dia e 116,3 a $138,0 \mathrm{~kg}$ para $\mathrm{PN}$, GDP e PDP, respectivamente.

O GNP e o PDP foram influenciados $(\mathrm{P}<0,05)$ pela raça da vaca, tendo os bezerros filhos de vacas Braford apresentado maiores GNP $(0,910 \mathrm{~kg} / \mathrm{dia})$ e maiores PDP $(129,3 \mathrm{~kg})$ em relação aos bezerros filhos de vacas Hereford $(0,811 \mathrm{~kg} /$ dia e $119,4 \mathrm{~kg})$.

Segundo Cartwright (1976), o ganho de peso dos bezerros até o desmame e o peso ao desmame depende da quantidade de leite e outros alimentos disponíveis, e da capacidade de crescimento do próprio terneiro. Lamond (1976) relata ganhos de peso pré-desmame de bezerros filhos de vacas cruza Brahman x Shorthorn de $0,645 \mathrm{~kg} / \mathrm{dia}$, enquanto aqueles bezerros filhos de vacas Shorthorn ganharam $0,236 \mathrm{~kg} /$ dia. 
Barcellos \& Lobato (1992a) avaliaram o ganho de peso pré-desmama de bezerros Hereford e Braford com $50 ; 75 ; 87,5$ e $37,5 \%$ de sangue taurino no genótipo, e observaram que os maiores ganhos de peso foram apresentados pelos bezerros com $75 \%$ de sangue taurino, ou seja, filhos de vacas $1 / 2$ sangue, com heterose máxima para produção de leite (Cartwright, 1976).

A data de nascimento não teve efeito $(\mathrm{P}>0,05)$ sobre o PAJ180 dos terneiros (Tabela 2). Isto está associado, provavelmente, às elevadas disponibilidades de forragem durante toda a estação de parição, influenciando o nível alimentar das vacas e a produção de leite das mesmas.

De acordo com a análise de variância, observou-se efeito da idade de desmame $(\mathrm{P}<0,01)$ e da interação tratamento $\mathrm{x}$ raça $(\mathrm{P}<0,05)$ sobre o GMD do nascimento ao desmame convencional, em 06.03.01 (GMD180), não havendo efeito $(P>0,05)$ da interação tratamento $\mathrm{x}$ idade de desmame. Conseqüentemente, os mesmos efeitos isolados de idade de desmame e da interação tratamento $\mathrm{x}$ raça da vaca, também foram observados para os pesos ajustados aos 180 dias (PAJ180). As médias ajustadas para GMD180 e PAJ180 estão na Tabela 2.

Bezerros machos tiveram maior GMD180 $(\mathrm{P}<0,05)$ que as fêmeas $(0,887 \mathrm{~kg} / \mathrm{dia}$ vs. $0,820 \mathrm{~kg} / \mathrm{dia})$ e foram $8,68 \%$ mais pesados aos 180 dias $(\mathrm{P}<0,01)$ em relação às fêmeas (196,1 kg vs. 180,51kg). Esses resultados estão de acordo com os resultados obtidos por vários autores, que também observaram maiores pesos ao desmame para os machos (Moraes \& Lobato, 1993; Lobato et al., 1998; Simeone \& Lobato, 1998).

Os bezerros filhos de vacas Braford submetidas à carga animal em campo nativo de $0,8 \mathrm{EV} /$ ha tiveram maiores $(\mathrm{P}<0,05)$ GMD180 e, conseqüentemente, maiores $(\mathrm{P}<0,05$ ou $\mathrm{P}<0,01) \mathrm{PAJ} 180$ em relação aos bezerros filhos de vacas Braford na carga animal de $0,6 \mathrm{EV} / \mathrm{ha}$ e a bezerros filhos de vacas Hereford na carga animal de $0,8 \mathrm{EV} / \mathrm{ha}$, não diferindo $(\mathrm{P}>0,05)$ as

Tabela 1 - Número de bezerros (NB), peso ao nascer (PN), ganho médio diário de peso até o desmame precoce (GNP) e peso na data do desmame precoce (PDP), conforme os tratamentos, a raça da vaca e o sexo dos bezerros

Table 1 - Number of calves (NC), birth weight (BW), average daily weight gain until the early weaning (GEW) and live weight at the early weaning date (WEW), by treatment, breed of cow and sex of calves

\begin{tabular}{|c|c|c|c|c|}
\hline & $\begin{array}{l}\mathrm{NB} \\
\mathrm{NC}\end{array}$ & $\begin{array}{l}\mathrm{PN}(\mathrm{kg}) \\
B W(\mathrm{~kg})\end{array}$ & $\begin{array}{l}\mathrm{GNP}(\mathrm{kg} / \text { dia }) \\
G E W(\mathrm{~kg} / \text { day })\end{array}$ & $\begin{array}{l}\mathrm{PDP}(\mathrm{kg}) \\
W E W(\mathrm{~kg})\end{array}$ \\
\hline \multicolumn{5}{|c|}{$\begin{array}{c}\text { Tratamento } \\
\text { Treatment }^{1}\end{array}$} \\
\hline $\mathrm{T} 1$ & 32 & $34,9 \mathrm{a}$ & $0,820 \mathrm{a}$ & $119,8 \mathrm{a}$ \\
\hline $\mathrm{T} 2$ & 32 & $34,4 \mathrm{a}$ & $0,904 \mathrm{a}$ & $127,1 \mathrm{a}$ \\
\hline $\mathrm{T} 3$ & 28 & $34,4 \mathrm{a}$ & $0,853 \mathrm{a}$ & $126,1 \mathrm{a}$ \\
\hline \multicolumn{5}{|l|}{$\begin{array}{l}\operatorname{Sexo}^{2} \\
\operatorname{Sex}^{2}\end{array}$} \\
\hline $\begin{array}{c}\text { Machos } \\
\text { Males }\end{array}$ & 32 & $36,3 \mathrm{~A}$ & 0,879 a & $128,1 \mathrm{a}$ \\
\hline Fêmeas & 60 & $32,8 \mathrm{~B}$ & 0,839 a & $120,4 \mathrm{a}$ \\
\hline \multicolumn{5}{|c|}{ Females } \\
\hline \multicolumn{5}{|c|}{ Raça da vaca ${ }^{2}$} \\
\hline \multicolumn{5}{|c|}{ Breed of cow ${ }^{2}$} \\
\hline Braford & 46 & 35,0 a & $0,910 \mathrm{a}$ & $129,3 \mathrm{a}$ \\
\hline Hereford & 46 & $34,1 \mathrm{a}$ & $0,811 \mathrm{~b}$ & $119,4 \mathrm{~b}$ \\
\hline $\mathrm{CV}^{3}(\%)$ & & 12,0 & 22,4 & 17,1 \\
\hline
\end{tabular}

1 Médias seguidas pela mesma letra minúscula (a), na coluna, não diferem entre si pelo teste $F$ $(P>0,05)$.

${ }_{1}^{1}$ Means followed by the same small letter (a), in the column, do not differ $(P>.05)$ by F-test.

2 Médias seguidas por letras minúsculas diferentes $(a, b)$ ou por letras maiúsculas diferentes (A,

$B)$, na coluna, diferem entre si pelo teste $F(P<0,05$ ou $P<0,01$, respectivamente).

2 Means followed by different small letters $(a, b)$ or by different capital letters $(A, B)$, in the column, are different by F-test $(P<.05$ or $P<.01$, respectively).

${ }^{3}$ Coeficiente de variação.

${ }^{3}$ Coefficient of variation.

R. Bras. Zootec., v.33, n.2, p.426-433, 2004 
últimas entre si. Os bezerros filhos de vacas de ambas as raças no tratamento de pastagem melhorada por 80 dias pós-parto e após carga animal de $320 \mathrm{~kg}$ $\mathrm{PV} /$ ha em campo nativo e filhos de vacas Hereford no tratamento de carga animal de $240 \mathrm{~kg}$ PV/ha em campo nativo não se diferenciaram ( $\mathrm{P}>0,05)$ (Tabela 2 ). Os maiores PAJ180 e GMD180 dos bezerros filhos de vacas Braford no T2 parecem indicar a manifestação de heterose materna, ou seja, quando submetidas à maior carga animal em campo nativo, onde a disponibilidade forrageira foi mais baixa, as vacas Braford expressaram heterose com maior produção de leite. Os menores GMD180 e PAJ180 dos bezerros filhos de vacas Hereford submetidas à mesma carga animal em campo nativo reforçam essa hipótese. Barcellos \& Lobato (1992a, b) observaram efeitos significativos de diferentes genótipos de bezerros filhos de vacas
Hereford e Braford com diferentes percentuais de sangue zebuíno e nascidos na primavera, no peso vivo dos bezerros ajustado aos 205 dias e no ganho médio diário aos 205 dias.

Os bezerros do DC (180 dias) foram $(\mathrm{P}<0,01)$ mais pesados $(197,2 \mathrm{~kg})$ e tiveram maiores $(\mathrm{P}<0,01)$ ganhos médios diários $(0,909 \mathrm{~kg} / \mathrm{dia})$ em relação aos bezerros submetidos à DP $(174,6 \mathrm{~kg}$ e 0,778 kg/dia, respectivamente; Tabela 2). Esses resultados podem ser explicados pelo efeito da amamentação sobre o ganho de peso dos bezerros até o desmame. Segundo Rovira (1996), cerca de 50\% da variação no peso ao desmame dos bezerros com seis meses de idade devem-se à variação no consumo de leite. Nas primeiras semanas de vida, os bezerros consomem semanalmente mais quilos de leite que seu próprio peso vivo, com oito semanas de vida consomem cerca

Tabela 2 - Ganho médio diário do nascimento até a idade do desmame convencional (GMD180) e peso ajustado aos 180 dias (PAJ180) conforme os tratamentos, sexo e idade de desmame

Table 2 - Average daily gain from birth to the age of conventional weaning and adjusted live weight at 180 days by treatment, sex, and weaning age

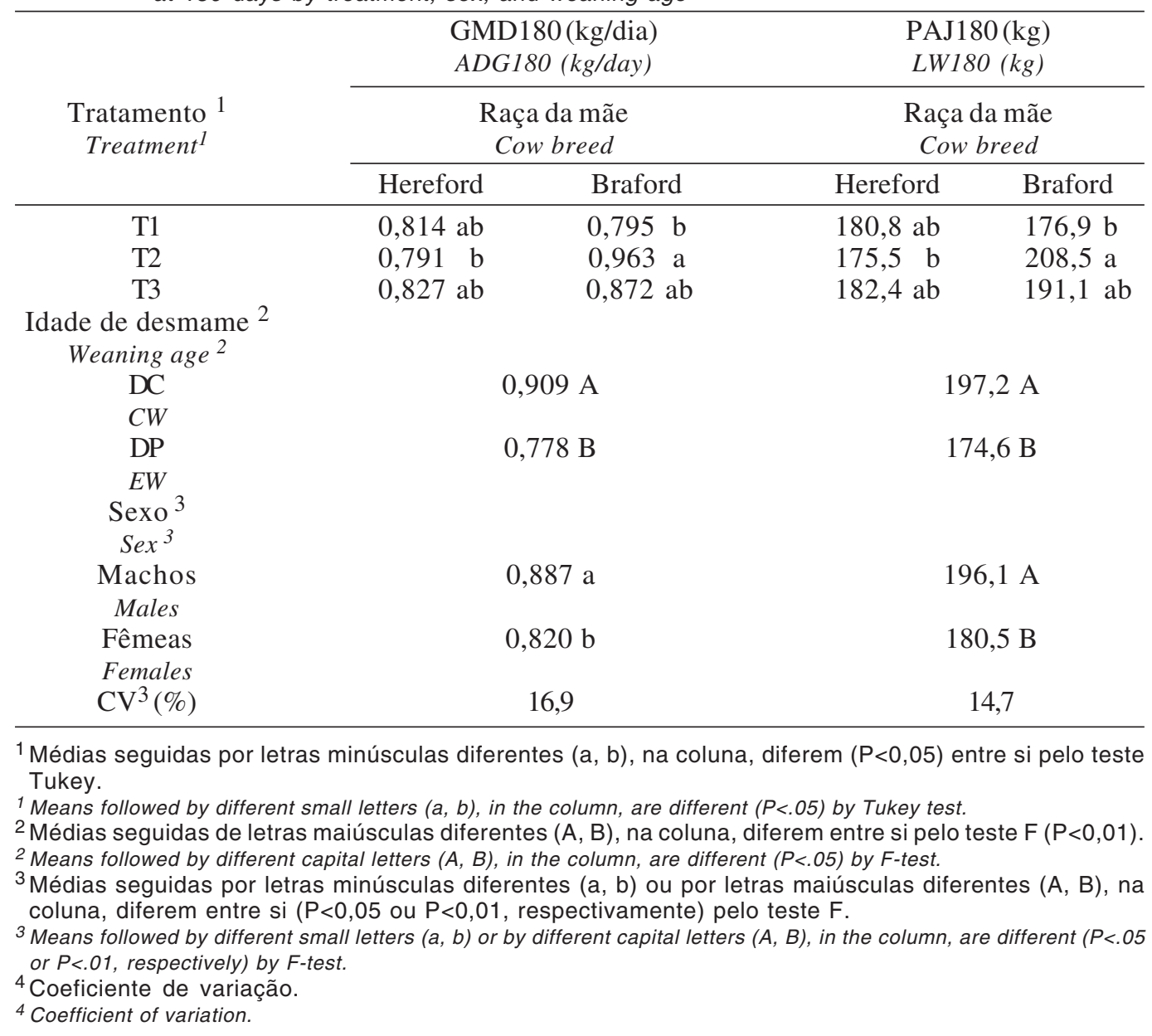

R. Bras. Zootec., v.33, n.2, p.426-433, 2004 
de $70 \%$ do seu peso vivo e com 12 semanas de vida, ou três meses de idade, têm um consumo de $50 \%$ do seu peso vivo em leite (Walker, 1963, citado por Rovira, 1996).

Embora os bezerros do DP tenham tido acesso a uma pastagem melhorada constituída fundamentalmente por cornichão, além da suplementação com concentrado, a interrupção abrupta da amamentação causou redução de ganho de peso inicialmente e, conseqüentemente, menor GMD180 e PAJ180. Pressupondo consumo médio de forragem por parte dos bezerros de $3 \%$ do seu peso vivo, a ingestão de nutrientes foi insuficiente para atender às exigências desse animais, os quais vinham consumindo $50 \%$ do seu peso vivo de um alimento de altíssima digestibilidade, quando comparado com qualquer forragem.

Os fatores estudados, tratamentos e idade de desmame, não influenciaram $(\mathrm{P}>0,05)$ o GMD (Tabela 3) desde a data do desmame convencional (06.03.01) aos 365 dias de idade (GMD365), bem como o peso vivo ajustado aos 365 dias de idade (PAJ365).

Com relação às interações duplas e tripla para as variáveis GMD365 e PAJ365, não foram observados os seus efeitos $(\mathrm{P}>0,05)$. Os valores variaram de 0,246 a 0,450 kg/dia e 220,4 a 276,0 kg para GMD365 e PAJ365, respectivamente.

O sexo dos bezerros foi fonte $(\mathrm{P}<0,01)$ de variação para GMD365 e PAJ365. Os bezerros machos tiveram maiores GMD $(0,552 \mathrm{~kg} / \mathrm{dia})$ em relação às fêmeas $(0,258 \mathrm{~kg} / \mathrm{dia})$ e foram mais pesados aos 365 dias de idade $(298,7 \mathrm{~kg}$ vs. $228,4 \mathrm{~kg})$. Simeone \& Lobato (1998) também observaram ser os machos mais pesados aos 365 dias de idade em relação às

Tabela 3 - Ganho médio diário do desmame a idade convencional aos 365 dias (GMD365) e peso vivo ajustado aos 365 dias de idade (PAJ365) conforme os tratamentos, a idade de desmame e o sexo

Table 3 - Average daily gain from the age at conventional weaning to 365 days (AGD365) and adjusted live weight at 365 days (LW365) by treatment, weaning age, and sex

\begin{tabular}{|c|c|c|}
\hline & $\begin{array}{l}\text { GMD365 (kg/dia) } \\
\text { ADG365 (kg/day) }\end{array}$ & $\begin{array}{l}\text { PAJ365 (kg) } \\
L W 365(\mathrm{~kg})\end{array}$ \\
\hline \multicolumn{3}{|c|}{ Tratamento 1} \\
\hline \multicolumn{3}{|l|}{ Treatment ${ }^{1}$} \\
\hline $\mathrm{T} 1$ & 0,340 a & 241,9 a \\
\hline $\mathrm{T} 2$ & $0,387 \mathrm{a}$ & $263,8 \mathrm{a}$ \\
\hline $\mathrm{T} 3$ & $0,355 \mathrm{a}$ & $252,5 \mathrm{a}$ \\
\hline \multicolumn{3}{|c|}{ Idade de desmame $^{1}$} \\
\hline \multicolumn{3}{|c|}{ Weaning age ${ }^{1}$} \\
\hline $\mathrm{DC}$ & $0,336 \mathrm{a}$ & 258,8 a \\
\hline \multicolumn{3}{|l|}{$C W$} \\
\hline DP & $0,384 \mathrm{a}$ & 246,6 a \\
\hline \multicolumn{3}{|l|}{$E W$} \\
\hline \multicolumn{3}{|l|}{ Sexo $^{2}$} \\
\hline \multicolumn{3}{|l|}{ Sex ${ }^{2}$} \\
\hline Machos & $0,552 \mathrm{~A}$ & 298,7 A \\
\hline \multicolumn{3}{|l|}{ Males } \\
\hline Fêmeas & $0,258 \mathrm{~B}$ & $228,4 \mathrm{~B}$ \\
\hline \multicolumn{3}{|l|}{ Females } \\
\hline $\mathrm{CV}^{3}(\%)$ & 18,6 & 17,0 \\
\hline $\begin{array}{l}{ }^{1} \text { Médias seguida } \\
\text { ( } P>0,05 \text { ) entre } \\
1 \text { Means followed } b \\
\text { by F-test. } \\
2 \text { Médias seguida } \\
\text { diferem ( } P<0,01 \\
{ }^{2} \text { Means followed by } \\
\text { by F-test. } \\
{ }^{3} \text { Coeficiente de } \\
{ }^{3} \text { Coefficient of vari }\end{array}$ & $\begin{array}{l}\text { a letra minúscula (a), } \\
\mathrm{F} \text {. } \\
\text { all letter (a), in the colum } \\
\text { maiúsculas diferent } \\
\text { to teste } \mathrm{F} \text {. } \\
\text { tal letters }(A, B) \text {, in the co }\end{array}$ & $\begin{array}{l}\text { na, não diferem } \\
\text { different }(P>.05) \\
\text { B), na coluna, } \\
\text { different }(P<.01)\end{array}$ \\
\hline
\end{tabular}

R. Bras. Zootec., v.33, n.2, p.426-433, 2004 
fêmeas. Na Tabela 3, estão as médias para GMD365 e PAJ365 conforme os tratamentos, a idade de desmame e o sexo dos bezerros.

O PAJ180, incluído como covariável no modelo, foi fonte $(\mathrm{P}<0,01)$ de variação para PAJ365. Para cada quilo de peso vivo a mais no PAJ180 houve aumento de 1,05 kg no PAJ365. Este efeito demonstra a importância de se proporcionarem adequados níveis de forragem para vacas amamentando, permitindo que as mesmas desmamem bezerros mais pesados, visando reduzir a idade de abate dos machos e a idade ao primeiro acasalamento das fêmeas.

Embora os bezerros do DP tenham apresentado GMD pré-desmame e pesos ao desmame ajustados aos 180 dias significativamente inferiores aos bezerros do DC, essa diferença não foi observada no peso e GMD ajustado aos 365 dias de idade $(\mathrm{P}>0,05)$. Esses resultados discordam da maioria dos trabalhos realizados no Rio Grande do Sul com bezerros submetidos a diferentes idades de desmama e recriados em campo nativo (Simeone \& Lobato, 1998; Lobato et al., 1999). Marques et al. (2000) compararam o desempenho de bezerros desmamados aos 91 (DP) e 170 (DC) dias de idade, suplementados ou não durante o primeiro inverno. Os bezerros do DP tiveram pesos vivos e GMD até um ano de idade inferiores $(\mathrm{P}<0,05)$ aos bezerros DC. Porém, os bezerros do DP, quando suplementados no inverno, não diferiram no peso vivo e GMD $(\mathrm{P}>0,05)$ até um ano de idade. Albospino \& Lobato (1994) compararam o desempenho de bezerros desmamados aos 100 (S1) e 150 (S2) dias de idade, onde os primeiros tiveram acesso, após o desmame, a potreiros com azevém e trevo vesiculoso, totalmente secos, diferidos por 60 dias para produção de sementes e ressemeadura natural, com forte predominância de capim-bermuda (Cynodon dactilum). Após o desmame definitivo, todos os bezerros foram manejados em um potreiro de campo nativo diferido até final de junho. Após esse período, os animais foram manejados em pastagem melhorada durante todo o inverno. Os pesos vivos dos bezerros e os GMD até um ano de idade não diferiram entre idades de desmame, sendo 196,6 kg para S1 e 194,3 kg para S2, e os ganhos de peso de $0,560 \mathrm{~kg} / \mathrm{dia}$ e $0,546 \mathrm{~kg} / \mathrm{dia}$ para $\mathrm{S} 1$ e $\mathrm{S} 2$, respectivamente.

Esses resultados indicam que o nível alimentar pós-desmame é de extrema importância, independentemente do peso ao desmame. Os animais desmamados precocemente, quando têm acesso à forragens de alta qualidade após o desmame, podem compensar o menor peso inicial durante a fase entre o desmame precoce e o desmame definitivo, sempre que o nível alimentar permita tal compensação. Fica clara também a necessidade de fornecer um nível alimentar adequado durante a recria, fundamental para não diminuir os possíveis benefícios na reprodução advindos da desmama precoce na fase de cria.

\section{Conclusões}

Bezerros filhos de vacas Braford têm ganhos de peso e peso vivo aos 100 dias de idade superior aos bezerros filhos de vacas Hereford.

Bezerros filhos de vacas Braford, em condições de cargas animais altas $(0,8 \mathrm{EV} / \mathrm{ha}) \mathrm{e}$, conseqüentemente, menores níveis de oferta de forragem, têm desenvolvimento pré-desmama aos 180 dias superior aos bezerros filhos de vacas Hereford.

O manejo pós-parto das vacas não influenciou o desenvolvimento dos bezerros até os 180 e até os 365 dias, indicando que, quando a disponibilidade forrageira é adequada, a carga animal ou a utilização de pastagem melhorada no pós-parto têm pequena influência sobre a produção de leite das vacas.

Bezerros desmamados em média aos 100 dias de idade têm desenvolvimento até os 12 meses semelhante ao dos bezerros desmamados aos 180 dias de idade.

\section{Literatura Citada}

ALBOSPINO, B.H.J.C.; LOBATO, J.F.P. Efeitos do desmame precoce de bezerros no desempenho até os 24-26 meses de idade. Revista Brasileira de Zootecnia, v.23, n.4, p.565-575, 1994.

BARCELLOS, J.O.J.; LOBATO, J.F.P. Efeitos da época de nascimento no desenvolvimento de bezerros Hereford e suas cruzas. I.Peso ao nascer e ganho médio diário prédesmama. Revista Brasileira de Zootecnia, v.21, n.1, p.137-149, 1992a.

BARCELLOS, J.O.J.; LOBATO, J.F.P. Efeitos da época de nascimento no desenvolvimento de bezerros Hereford e suas cruzas. II.Pesos ao desmame, ano e sobreano. Revista Brasileira de Zootecnia, v.21, n.1, p.151-157, 1992b.

CARTWRIGHT, T.C. Comparacion entre vacas $\mathrm{F}_{1}$ con las de raza pura y otras cruzas. In: KOGER, M.; CUNHA, T.J.; WARNICK, A.C. (Eds.) Cruzamientos en ganado vacuno de carne. Montevideo: Hemisferio Sur, 1976. p.62-80.

COCIMANO, M.; LANGE, A.; MENVIELLE, E. et.al. Equivalências ganaderas para vacunos de carne y ovinos. 4.ed. CREA, 1983. 32p. (Estudios y metodos, 1)

COSTA, E.C.; RESTLE, J.; BRONDANI, I.L. et. al. Composição física da carcaça, qualidade da carne e conteúdo de colesterol no músculo Longissimus dorsi de novilhos Red Angus superprecoces, terminados em confinamento e abati-

\section{R. Bras. Zootec., v.33, n.2, p.426-433, 2004}


dos com diferentes pesos. Revista Brasileira de Zootecnia, v.31, n.1 (supl.), p.417-428, 2002.

CUNDIFF, L.V.; GREGORY, K.E.; SCHWULST, F.J. et al. Effects of heterosis on maternal performance and milk production in Hereford, Angus and Shorthorn cattle. Journal of Animal Science, v.38, n.4, p.728-745, 1974.

ESPASANDIN, A.C.; PACKER, I.U.; ALENCAR, M.M. et. al. Estimativas de parâmetros genéticos para a produção de leite em bovinos de corte. In: REUNIÃO ANUAL DA SOCIEDADE BRASILEIRA DE ZOOTECNIA, 38., 2001, Piracicaba. Anais ... Piracicaba: Sociedade Brasileira de Zootecnia, 2001. 5p.

GREGORY, K.E.; CUNDIFF, L.V.; KOCH, R.M. Breed effects and heterosis in advanced generations of composite populations for reproduction and maternal traits of beef cattle. Journal of Animal Science, v.70, n.3, p.656-672, 1992.

HOLLAND, B.J.M.; MULLANEY, P.D.; HOPKINS, I.R. Breed and enviromental factors affecting birth weight in Victorian beef cattle. Australian Journal of Experimental Agriculture and Animal Husbandry, v.17, n.84, p.5-9, 1977.

LAMOND, D.R. Indices de reproducción y destete de ganado de raza pura y cruza en Australia y Nueva Zelandia. In: KOGER, M.; CUNHA, T.J.; WARNICK, A.C. (Eds.) Cruzamientos en ganado vacuno de carne. Montevideo: Hemisferio Sur, 1976. p.190-201.

LOBATO, J.F.P.; PEREIRA NETO, O.A.; MÜLLER, A. et al. Efeito da desmama precoce sobre o desenvolvimento dos bezerros até um ano de idade. In: REUNIÃO ANUAL DA SOCIEDADE BRASILEIRA DE ZOOTECNIA, 36., 1999, Porto Alegre. Anais ... Porto Alegre: Sociedade Brasileira de Zootecnia, 1999. 3p.

LOBATO, J.F.P.; ZANOTTA JR., R.L.D.; PEREIRA NETO, O A. Efeitos das dietas pré e pós-parto de vacas primíparas sobre o desenvolvimento dos bezerros. Revista Brasileira de Zootecnia, v.27, n.5, p.863-867, 1998.

MARQUES, L.P.A.; LOBATO, J.F.P.; SCHENKEL, F.S. Efeito da idade de desmame e suplementação no desenvolvimento de novilhos de corte. In: REUNIÃO ANUAL DA SOCIEDADE BRASILEIRA DE ZOOTECNIA, 38., 2001, Piracicaba. Anais... Piracicaba: Sociedade Brasileira de Zootecnia, 2001a. p.415.

MARQUES, L.P.A.; LOBATO, J.F.P.; SCHENKEL, F.S. Efeito da idade de desmame e suplementação no desenvolvimento de novilhas de corte. In: REUNIÃO ANUAL DA SOCIEDADE BRASILEIRA DE ZOOTECNIA, 38., 2001, Piracicaba. Anais... Piracicaba: Sociedade Brasileira de Zootecnia, 2001b. p.416.
MARQUES, L.P.A.; LOBATO, J.F.P.; SCHENKEL, F.S. Época de desmame e suplementação no desenvolvimento de terneiros de corte. In: REUNIÃO ANUAL DA SOCIEDADE BRASILEIRA DE ZOOTECNIA, 37., 2000, Viçosa, MG. Anais... Viçosa, MG: Sociedade Brasileira de Zootecnia, 2000.

MOORE, C.P.; ROCHA, C.M.C.; SAUERESSIG, M.G. Efeito de níveis de energia pós-parto e idade à desmama sobre o desenvolvimento de bezerros até 24 meses de idade. In: REUNIÃO ANUAL DA SOCIEDADE BRASILEIRA DE ZOOTECNIA, 20., 1983, Pelotas. Anais... Pelotas: Sociedade Brasileira de Zootecnia, 1983. p.137.

MORAES, A.A.S.; LOBATO, J.F.P. Efeito de duas idades de desmame no desenvolvimento de terneiros de corte. Revista Brasileira de Zootecnia, v.22, n.6, p.885-892, 1993.

QUADROS, S.A.F.; LOBATO, J.F.P. Efeitos da lotação animal na produção de leite de vacas de corte primíparas e no desenvolvimento de seus bezerros. Revista Brasileira de Zootecnia, v.26, n.1, p.27-33, 1997.

RIBEIRO, A.M.L.; LOBATO, J.F.P. Produtividade e eficiência reprodutiva de três grupos raciais de novilhas de corte. II.Desenvolvimento da progênie até o desmame. Revista Brasileira de Zootecnia, v.17, n.6, p.509-515, 1988.

ROVIRA, J.M. Manejo nutritivo de los rodeo de cría en pastoreo. Montivideo: Editorial Hemisferio Sur, 1996. 287p.

SAKAGUTI, E.S.; SILVA, M.A.; MARTINS, E.N. et. al. Efeito da idade da mãe e do sexo do animal sobre o peso corporal de bovinos Tabapuã em diferentes idades. In: REUNIÃO ANUAL DA SOCIEDADE BRASILEIRA DE ZOOTECNIA, 38., 2001, Piracicaba. Anais ... Piracicaba: Sociedade Brasileira de Zootecnia, 2001. p.552.

SIMEONE, A.; LOBATO, J. F. P. Efeitos da carga animal em campo nativo e do controle da amamentação no desenvolvimento de bezerros mestiços até um ano de idade. Revista Brasileira de Zootecnia, v.27, n.1, p.179-185, 1998. 